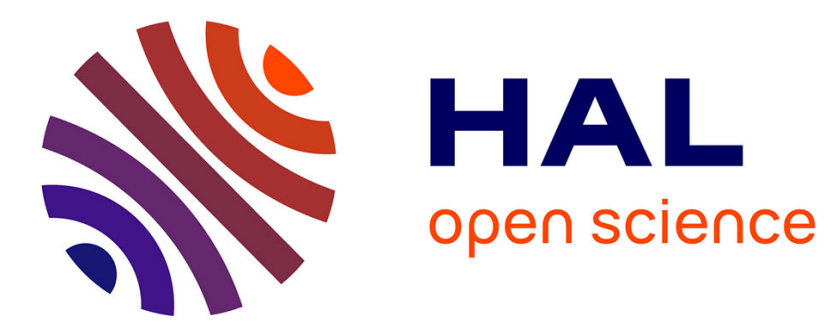

\title{
Structural Characterization of Thin Films and Multilayer Structures
}

K. Temst, M. van Bael, M. Baert, E. Rosseel, V. Bruyndoncx, C. Strunk, G. Verbanck, K. Mae, C. van Haesendonck, V. Moshchalkov, et al.

\section{- To cite this version:}

K. Temst, M. van Bael, M. Baert, E. Rosseel, V. Bruyndoncx, et al.. Structural Characterization of Thin Films and Multilayer Structures. Journal de Physique IV Proceedings, 1996, 06 (C3), pp.C3265-C3-270. 10.1051/jp4:1996340 . jpa-00254258

\section{HAL Id: jpa-00254258 https://hal.science/jpa-00254258}

Submitted on 1 Jan 1996

HAL is a multi-disciplinary open access archive for the deposit and dissemination of scientific research documents, whether they are published or not. The documents may come from teaching and research institutions in France or abroad, or from public or private research centers.
L'archive ouverte pluridisciplinaire HAL, est destinée au dépôt et à la diffusion de documents scientifiques de niveau recherche, publiés ou non, émanant des établissements d'enseignement et de recherche français ou étrangers, des laboratoires publics ou privés. 


\title{
Structural Characterization of Thin Films and Multilayer Structures
}

K. Temst, M.J. Van Bael, M. Baert, E. Rosseel, V. Bruyndoncx, C. Strunk, G. Verbanck, K. Mae, C. Van Haesendonck, V.V. Moshchalkov, Y. Bruynseraede, R. Jonckheere*, D.G. de Groot**, N. Koeman** and R. Griessen**

Laboratorium voor Vaste-Stoffysica en Magnetisme, Katholieke Universiteit Leuven, Celestijnenlaan 200 D, 3001 Leuven, Belgium

* IMEC vzw, Kapeldreef 75, 3001 Leuven, Belgium

** Faculteit Natuurkunde en Sterrenkunde, Vrije Universiteit Amsterdam, De Boelelaan 1081, 1081 HV

Amsterdam, The Netherlands

\begin{abstract}
Atomic force microscopy has been used to study the structure, down to the nanoscale, of thin-film and multilayer samples. Due to the fact that atomic force microscopy is not restricted to conducting surfaces, it is possible to image mesoscopic rings, lines, and more complex structures deposited on insulating substrates. X-ray diffraction provides access to the internal structure of the layers, interface roughness and the lateral periodicity of antidot lattices. Comparison of atomic force microscopy and x-ray diffraction on molecular beam epitaxy grown $\mathrm{Nb} / \mathrm{Cu}$ multilayers shows that high-angle diffraction averages over a lateral length which is in good agreement with the typical grain size.
\end{abstract}

\section{INTRODUCTION}

A rapidly increasing number of research topics and applications in science and engineering rely on thin films, superlattice growth, and patterning down to the submicrometer scale. In order to understand the physical properties of such nanostructures, a thorough and detailed structural characterization is required. Scanning probe microscopy is a very powerful tool for studying the surface structure of such samples. $\mathrm{X}$-ray diffraction (XRD) techniques allow us to probe the internal structure of the layers and the quality of the interfaces between the different materials in a multilayer. Combining the two techniques, information on the lateral correlation lengths of surface and interface roughness can be obtained.

\section{EXPERIMENTAL RESULTS}

\subsection{Atomic force microscopy}

Atomic force microscopy (AFM) has established itself as a very versatile tool within the family of scanning probe microscopy techniques, due to the fact that non-conducting surfaces can be imaged. This is especially important in the study of small samples deposited on insulating substrates. A drawback is that tip-shape effects can be more pronounced and that atomic-scale resolution is harder to achieve than in scanning tunneling microscopy. The AFM experiments shown in this paper were performed with a Digital Instruments NanoScope III apparatus, using $\mathrm{Si}_{3} \mathrm{~N}_{4}$ tips on a cantilever with a $0.12 \mathrm{~N} / \mathrm{m}$ spring constant. All measurements were carried out in the contact mode.

Figure 1 (a) shows an AFM picture of a mesoscopic Al loop which was prepared by thermal evaporation of the $\mathrm{Al}$ layer in a resist pattern defined by electron beam lithography, followed by a standard lift-off procedure. These superconducting Al loops show interesting new phenomena due to the interaction between transport and shielding currents [1]. Figure 1 (b) shows a superconducting $\mathrm{Pb} / \mathrm{Ge}$ multilayer with a regular array of submicrometer-sized holes ('antidots'), produced in a similar way as the Al loops. The AFM pictures allow to inspect the structures for the presence of weak spots or cracks, which would influence the superconducting behaviour. The AFM characterization is complementary to that by scanning electron microscopy because it provides an independent measure of the linewidths and grain sizes, but it also yields a quantitative measure of the surface roughness. 

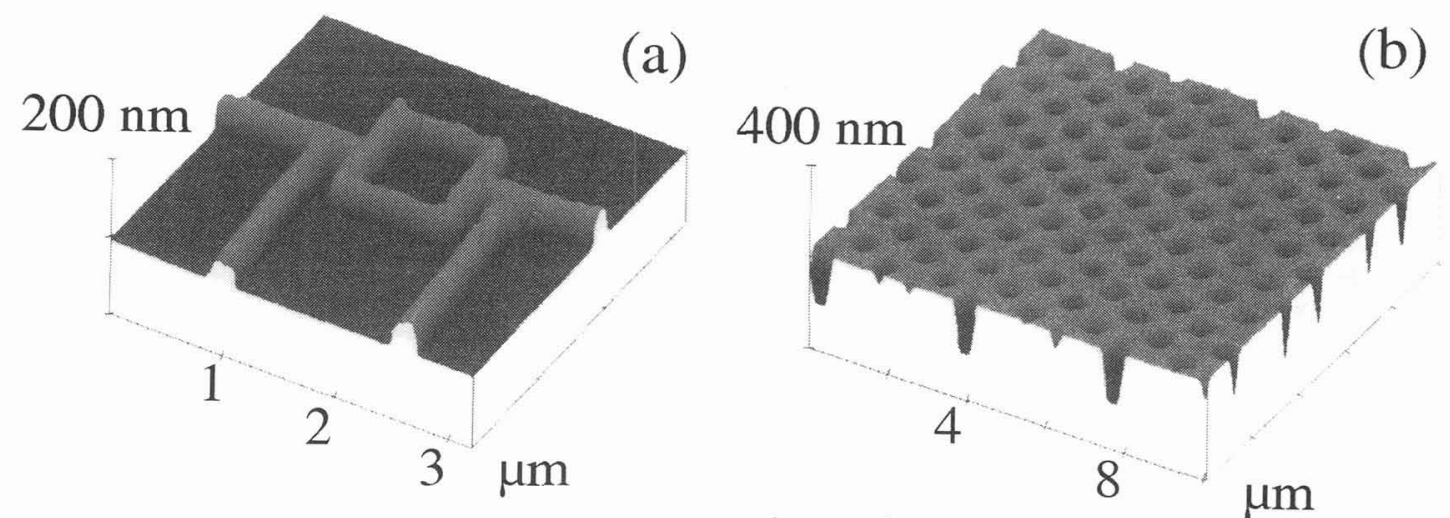

Figure 1: $A \overline{A F M}$ pictures of a mesocopic $\mathrm{Al}$ loop (a) and a $[\mathrm{Pb}(123 \AA) / \mathrm{Ge}(43 \AA)]_{3}$ multilayer with a lattice of antidots (b).

\subsection{Characterization combining atomic force microscopy and $x$-ray diffraction}

In this part we will present the structural characterization of superconducting thin films in which a regular array of submicrometer-sized holes ('antidots') is created by means of lithographic techniques. It has been shown before that the presence of these antidots leads to a number of new interesting features in the superconducting properties, notably a strong overall increase of the critical current density and additional peaks at those field values where a matching occurs between the flux line lattice and the antidots, where the flux lines are trapped [2].

Figure 1 (b) shows an AFM picture of a $[\mathrm{Pb}(123 \AA) / \mathrm{Ge}(43 \AA)]_{3}$ film with a square antidot lattice. The crystalline/amorphous $\mathrm{Pb} / \mathrm{Ge}$ multilayer is obtained by evaporation in a molecular beam epitaxy system. The antidots are obtained by a standard lift-off procedure. An atomic force microscopy study revealed that the surface roughness between the holes (which are spaced $1 \mu \mathrm{m}$ apart) is of the order of $10 \AA$ (all roughness values quoted in this paper are rms values). The AFM picture shows the regularity of the antidot pattern and the quality of the lift-off procedure.

These samples have also been characterized by $x$-ray diffraction experiments in which the specular and the diffuse scattering were recorded. The specular scans provide information about the periodicity of the multilayering, while the diffuse scans yield information about the lateral periodicity of the antidot lattice.
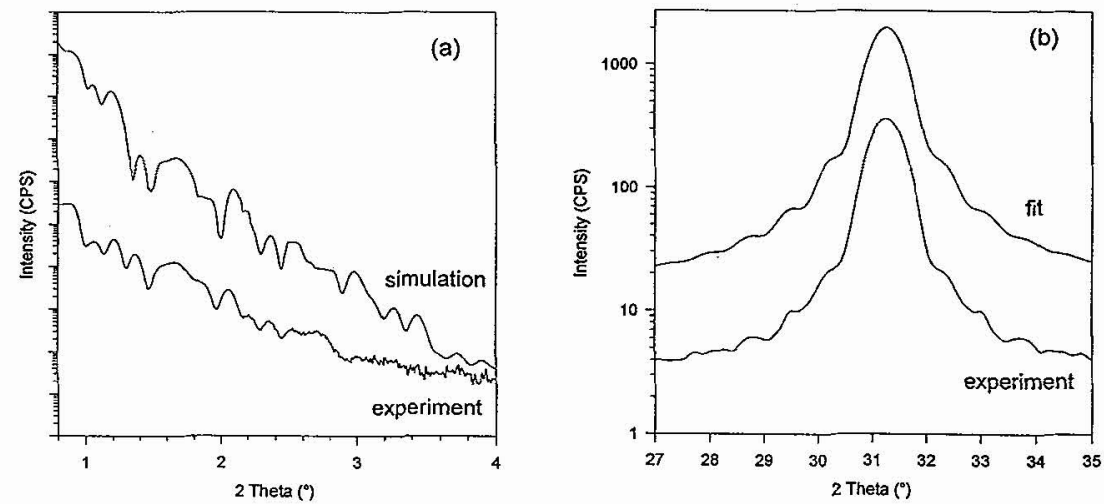

Figure 2: Low-angle (a) and high-angle (b) specular scattering of the $[\mathrm{Pb}(123 \AA) / \mathrm{Ge}(43 \AA)]_{3}$ multilayer with a lattice of antidots. The lower curves are the experimental data, the upper curves are calculated by the Suprex program.

The specular XRD data (shown in Fig. 2) have been analyzed using the Suprex modeling and fitting program [3], which uses a kinematical structure calculation for the high-angle spectra, while an optical model is used for the low-angle data. Imperfections in the multilayer are included by the introduction of a number of parameters. For crystalline layers, the roughness is included by assuming random variations in 
the number of monolayers in the crystalline layer. These fluctuations are, therefore, named discrete, i.e., 'quantized' in steps equal to the lattice spacing and are presumed to have a Gaussian distribution. Amorphous layers have a roughness which is modelled as continuously variable fluctuations of the layer thickness, again assumed to have a Gaussian distribution. Furthermore, it is assumed that there can be a fluctuation of the interface distance, i.e., the vertical distance between two dissimilar atoms at the interface between two layers. Suprex can perform simulations, as well as fittings to measured data.

Figure 2 (a) shows the low-angle specular scattering of the $[\mathrm{Pb}(123 \AA) / \mathrm{Ge}(43 \AA)]_{3}$ film with a square antidot lattice. The lower curve shows the experimental data, while the upper curve is a simulation using the Suprex program. Both the peak positions and the relative peak intensities can be well reproduced. Due to the fact that there are only three bilayers in this sample, the multilayer peaks are not easily distinguishable from the finite-size peaks, but nevertheless a detailed analysis proved to be possible, providing individual $\mathrm{Pb}$ and $\mathrm{Ge}$ thicknesses of $123 \AA$ and $43 \AA$, respectively. In the simulation an interface roughness of $12 \AA$ was assumed, in fair agreement with the roughness measured by AFM on a lateral length scale of a few thousand $\AA$. There is evidence for the existence of a thin interdiffused layer near the interfaces with a thickness of $1.5 \AA$ for a $\mathrm{Pb} / \mathrm{Ge}$ interface and $5 \AA$ for a Ge/Pb interface.

Figure 2 (b) shows the high-angle spectrum of the sample (lower curve) together with a fit to the data (upper curve). The high-angle spectrum of a $\mathrm{Pb} / \mathrm{Ge}$ multilayer typically shows an intense peak, flanked by a number of finite-size peaks. The periodicity of these finite-size peaks shows that the spectrum is actually created by the diffraction of a single crystalline layer (with an average thickness of $123 \AA$ ) and it does not contain any information on the multilayer superstructure. This is due to the fact that thickness fluctuations of the amorphous Ge layer restrict the crystalline coherence length to a single $\mathrm{Pb}$ layer [4]. We find $8.5 \AA$ (about three monolayers) of discrete layer thickness fluctuations of the Pb layers. This is slightly higher than what was found for a $\mathrm{Pb} / \mathrm{Ge}$ multilayer without patterning, which was evaporated simultaneously with the patterned one. This analysis shows that the crystalline structure of the Pb layers and the multilayer quality are only slightly affected by introducing the lattice of antidots.

The lateral periodicity of the antidot lattice was investigated by performing $\omega$-scans, in which the angle between the detector and the incoming $\mathrm{x}$-ray beam is kept constant, while the sample is rotated about an axis perpendicular to the plane of source and detector. In this configuration, the scattering vector has a fixed magnitude, but is tilted away from the perpendicular direction over an angle $\omega$, revealing the ratio of diffuse and specular scattering. For samples with a lateral diffraction grating additional maxima show up in the $\omega$-scan [5].

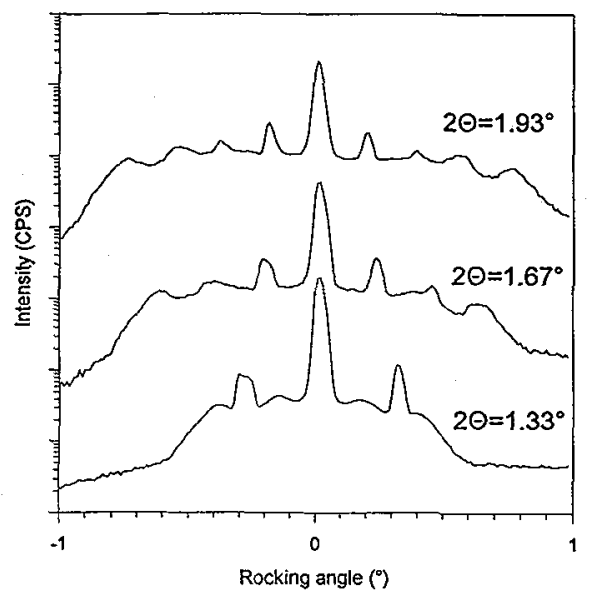

Figure 3: $\omega$-scans at three different values of $2 \theta$ on the $[\mathrm{Pb}(123 \AA) / \mathrm{Ge}(43 \AA)]_{3}$ sample with antidots.

Figure 3 shows the result of three $\omega$-scans performed at the indicated $2 \theta$ values where the sample was placed in such a way that the antidot lattice is rotated over $45^{\circ}$ with respect to the incoming X-ray beam. All spectra in Fig. 3 show a strong central peak, located at the position of the specular scattering, flanked by satellite peaks due to the lateral periodicity. More satellite peaks can be observed at higher $2 \theta$ values. 
From the position of the maxima a lateral periodicity of $1.4 \mu \mathrm{m}$ can be calculated, which corresponds to a distance $d=1 \mu \mathrm{m}$ between the antidots, in agreement with the AFM results.

\subsection{Roughness in $\mathrm{Nb} / \mathrm{Cu}$ multilayers studied by $\mathrm{XRD}$ and $\mathrm{AFM}$}

In section 2.2 we have used the Suprex model to obtain an estimate of the interface roughness for multilayered structures. This roughness can never be expressed by a single number, however, since it is always related to some sampling length measured in the interface or surface plane. A problem associated with determining the roughness from $x$-ray diffraction measurements is precisely that this lateral length scale is not known. AFM measurements provide the possibility to measure directly the roughness as a function of sampling length, like in similar experiments using scanning tunneling microscopy. A difficulty is that, unless the experiments is performed in situ during growth, only the outer surface of a sample can be probed.

For our study of $\mathrm{Nb} / \mathrm{Cu}$ multilayers we have combined XRD and AFM measurements to investigate the roughness as a function of the lateral length scale. The number of bilayers $(N)$ in the sample was varied, so that AFM gives also an idea about the internal interfaces, provided there occur no structural changes after the deposition of extra layers. The main purpose was to establish experimentally the lateral length scale over which the $\mathrm{x}$-ray beam averages. $\mathrm{Nb} / \mathrm{Cu}$ yields excellent multilayers, and the influence of layer thickness fluctuations on the high-angle XRD spectra has already been investigated [3].

$\mathrm{The} \mathrm{Nb} / \mathrm{Cu}$ multilayer samples were grown at room temperature by molecular beam epitaxy at a pressure of $10^{-9}$ Torr during the evaporation. The evaporation rates were $1 \AA / \mathrm{s}$ for both $\mathrm{Nb}$ and $\mathrm{Cu}$, and were controlled using quartz crystal monitors. As substrates sapphire crystals were used, which prior to the evaporation run were annealed at $300^{\circ} \mathrm{C}$. All samples had a $[\mathrm{Nb}(30 \AA) / \mathrm{Cu}(20 \AA)]_{N}$ structure with $N$ varying between 2 and 40 . From the XRD characterization it was found that all samples have the $\mathrm{Nb}(110)$ and $\mathrm{Cu}(111)$ planes parallel to the substrate. Within the plane of the layers the grains have random orientations, as could be deduced from asymmetric scattering experiments.
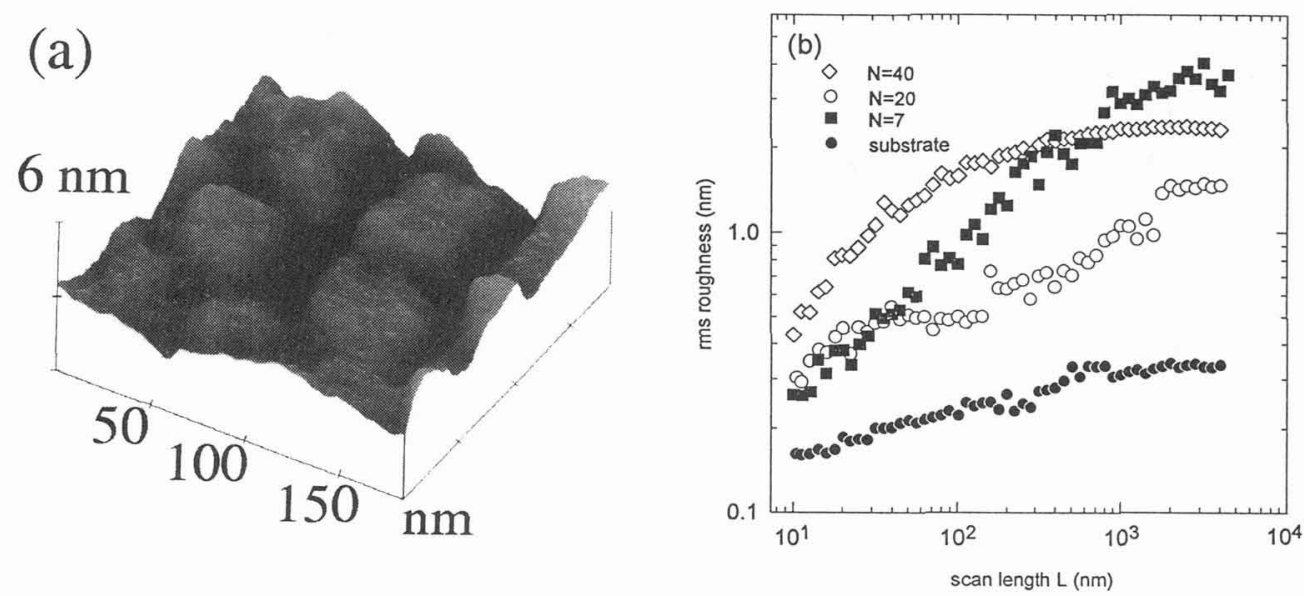

Figure 4: (a) AFM scan of the surface of the $\mathrm{Nb} / \mathrm{Cu}$ multilayer with $N=20$ bilayers, showing an area of $200 \mathrm{~nm}$ by $200 \mathrm{~nm}$; (b) Evolution of the rms roughness as a function of sampling length $L$, derived from the AFM measurements on the sapphire substrate and the $\mathrm{Nb} / \mathrm{Cu}$ multilayers with $N=7, N=20$, and $N=40$ bilayers.

Figure 4 (a) shows a typical AFM top view of the surface of the $N=20$ multilayer. This scan, covering an area of $200 \mathrm{~nm}$ by $200 \mathrm{~nm}$, allows us to estimate the average grain size, which is of the order of $45 \mathrm{~nm}$ and is independent of $N$. The grains have a smooth upper surface, but there are considerable height variations between neighbouring grains. 
The AFM data are analyzed by averaging the roughness of an area $L \times L$ for 15 different, randomly chosen positions on the sample surface in order to minimize the influence of local topographic variations. Figure 5 (b) shows this averaged rms roughness as a function of sampling length $L$ for the multilayers with $N=7, N=20$, and $N=40$. For $L<40 \mathrm{~nm}$ this averaged roughness is higher than the roughness measured on the grain top surface, due to the influence of the grain boundaries which create valleys in between adjacent grains. As a reference, the measured rms roughness of the sapphire substrate is also shown and ranges from about $0.15 \mathrm{~nm}$ at the shortest scan lengths (an area of $10 \times 10 \mathrm{~nm}^{2}$ ) to about 0.3 $\mathrm{nm}$ at the largest length scales (an area of $4 \times 4 \mu \mathrm{m}^{2}$ ).

For all $N$ the multilayer roughness is considerably larger than the substrate roughness, the enhancement being more pronounced for larger $N$. From our AFM experiments we also conclude that the rms roughness values for both very small and very large scan sizes are nearly independent of the total film thickness, i.e., the roughness appears to have a noncumulative character. The precise way in which the roughness grows with the scan length is, however, strongly dependent on the total multilayer thickness. The transition from a slow (as for the substrate and the $N=7$ film) to a very fast (as for the $N=40$ sample) increase of roughness can be interpreted as a decrease of the lateral correlation length.

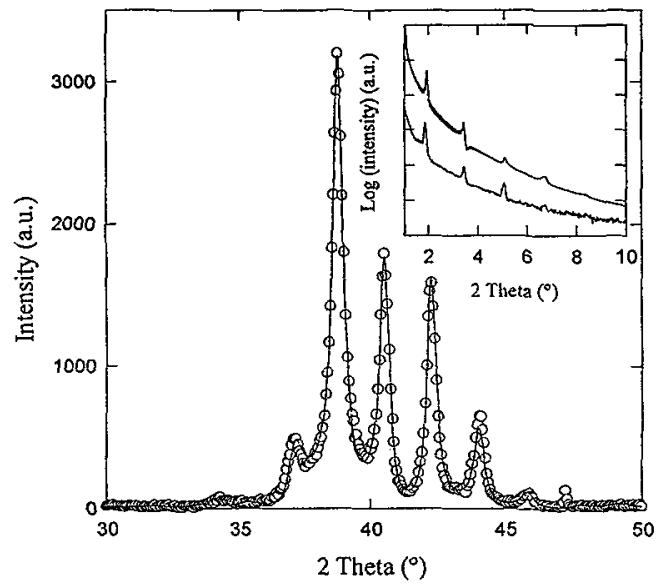

Figure 5: Fitting of the high-angle spectrum of the $\mathrm{Nb} / \mathrm{Cu}$ multilayer with $N=20$ bilayers. The dots are the experimental data, while the full line is the best fit. The inset shows a simulation (upper curve) of the experimental (lower curve) lowangle data of this sample. Both curves have been displaced for clarity.

Figure 5 shows the result of the Suprex fitting procedure for the $\mathrm{Nb} / \mathrm{Cu}$ multilayer with $\mathrm{N}=20$. An excellent agreement beteen the experiment and the model can be achieved using the following parameters: the lattice parameters for $\mathrm{Nb}$ and $\mathrm{Cu}$ are $d_{N b}=2.33 \AA$ and $d_{C u}=2.09 \AA$ respectively; the layer thickness fluctuations are equal to $\sigma_{N b}=0.17$ monolayers for $\mathrm{Nb}$ and $\sigma_{C u}=1.0$ monolayer for $\mathrm{Cu}$; the interface distance fluctuation width $\sigma_{c}$ is equal to $0.3 \AA$. The total rms roughness is then $\sigma_{t}=0.22 \mathrm{~nm}$ [6]. The inset of Fig. 5 shows the low-angle spectrum of the same multilayer, together with a simulation of this spectrum using an optical model and providing a roughness value $\sigma_{t}=0.5 \mathrm{~nm}$, i.e., considerably larger than the result obtained from the high-angle spectrum. A more detailed analysis of the low-angle spectra requires subtraction of the diffuse scattering at the specular condition in order to obtain the true specular scattering [7]. This procedure leads to a higher roughness value, implying that the result $\sigma_{t}=0.5 \mathrm{~nm}$ corresponds to a lower limit for the roughness. It should be noted here that comparable roughness values are obtained from fitting all the spectra of the varous samples. Within the error margin of these fittings (typically about $0.02 \mathrm{~nm}$ ) the roughness remains independent of the total thickness.

We can now compare the roughness values measured directly using the AFM with those obtained from fitting the XRD spectra. It should be stressed here that this comparison is not straightforward, because AFM provides information on the outer surface, while XRD makes an average over all the interfaces 
buried within the multilayer. There is, however, no obvious reason why in the $\mathrm{Nb} / \mathrm{Cu}$ system the outer surface would differ significantly from the underlying layers. The AFM surface roughness results are therefore believed to be indicative of the roughness of the interfaces and can be seen as a map which charts this roughness as a function of the lateral length scale. The high-angle XRD roughness values correspond to the surface roughness obtained from the AFM data for lateral length scales $L$ of about 30 $\mathrm{nm}$, i.e., close to the average grain size found in the AFM topographs. This remarkable agreement was observed in all samples studied. The simulations of the low-angle XRD spectra yielded higher roughness values which, according to the AFM data, agree with a lateral length of about $200 \mathrm{~nm}$ for the thinner multilayers. For the thickest multilayer $(N=40)$ this lateral length seems to be smaller, probably due to a loss of coherence in the multilayer stacking, as was also observed in $\mathrm{Nb} / \mathrm{Si}$ multilayers showing strong columnar growth [7]. As mentioned above, the lateral length scale obtained from the low-angle diffraction data only corresponds to a lower limit, since the removal of the diffuse scattering component can lead to still higher roughness values. This lateral length remains, however, much smaller than the saturation length encountered in the AFM experiments.

The different length scales over which the $\mathrm{x}$-ray beam is averaging for low-angle and high-angle diffraction may be understood as a difference in the crystalline coherence length, i.e., the crystalline volume which is scattering coherently. For high-angle diffraction, which is probing periodicities which are of the order of the interatomic distances, the coherently scattering volume may be limited to the size of a grain. Low-angle diffraction looks at larger periodicities and is, therefore, less sensitive to defects at the atomic scale. The crystalline coherence length can comprise several grains in that case and the roughness over a longer lateral length scale is sampled. This conclusion is very important for the XRD analysis of multilayer structures, because it shows that the roughnesses obtained from high-angle as well as low-angle XRD measurements have a limited lateral validity.

\section{CONCLUSIONS}

In conclusion, we have demonstrated the possibilities of scanning probe microscopy in the characterization of the surface of thin films and multilayered structures. X-ray diffraction provides access to the interior of the layers. Combining both techniques, new information can be extracted about lateral roughness correlation lengths and, hence, also about the validity of roughness parameters obtained from the analysis of the $\mathrm{x}$-ray diffraction data.

\section{Acknowledgments}

The authors thank J.P. Celis (MTM, K.U. Leuven) and his team for the use of the AFM facilities. This work has been financed by the Belgian Interuniversity Attraction Poles (IUAP) and the Flemish Concerted Action (GOA) programs, and by the Belgian National Fund for Scientific Research (NFWO).

\section{References}

[1] Moshchalkov V.V., Gielen L., Dhallé M., Van Haesendonck C., and Bruynseraede Y., Nature 361 (1993) 617

[2] Baert M., Metlushko V.V., Jonckheere R., Moshchalkov V.V., and Bruynseraede Y., Phys. Rev. Lett. 74 (1995) 3269

[3] Fullerton E., Schuller I.K., Vanderstraeten H., and Bruynseraede Y., Phys. Rev. B 45 (1992) 9292

[4] Neerinck D., Vanderstraeten H., Stockman L., Locquet J.-P., Bruynseraede Y., and Schuller I.K., J. Phys.: Condens. Matter 2 (1990) 4111

[5] Shen Q., Umbach C.C., Weselak B., and Blakely J.M., Phys. Rev. B 48 (1993) 17967

[6] Temst K., Van Bael M.J., Wuyts B., Van Haesendonck C., Bruynseraede Y., de Groot D.G., Koeman N., and Griessen R., Appl. Phys. Lett. 67 (1995) 3429

[7] Fullerton E.E., Pearson J., Sowers C.H., Bader S.D., Wu X.Z., and Sinha S.K., Phys. Rev. B 48 (1993) 17432 